\title{
Aislamiento y serotipificación de Salmonella sp. en estanques con Crocodylus intermedius y testudines cautivos en Villavicencio - Colombia
}

\author{
Isolation and serotyping of Salmonella sp. in ponds with captive \\ Crocodylus intermedius and testudines in Villavicencio - Colombia
}

Diana Pachón C, ${ }^{1}$ Mic. Agríc y Vet, Adriana Pulido V, ${ }^{1 *}$ M.Sc, , Carlos Moreno T, 2 MV.

${ }^{1}$ Pontificia Universidad Javeriana. Facultad de Ciencias. Departamento de Microbiología. Unidad de Investigaciones Agropecuarias - UNIDIA - Línea de epidemiología y salud animal. Bogotá - Colombia. ¿Universidad Nacional de Colombia. Facultad de Medicina Veterinaria y Zootecnia. Departamento de Ciencias para la Salud Animal. Laboratorio Clínico. Bogotá - Colombia. *Correspondencia: adriana. pulido@javeriana.edu.co

Recibido: Febrero de 2010; Aceptado: Enero de 2011.

\section{RESUMEN}

Objetivo. Determinar la presencia de microorganismos del género Salmonella sp. en el ambiente acuático de los ejemplares Crocodylus intermedius y testudines en la Estación de Biología Tropical Roberto Franco (EBTRF). Materiales y métodos. En este estudio se utilizó la metodología estándar para aislar e identificar microorganismos del género Salmonella sp., a partir de muestras de agua y sedimento de 52 estanques $\left(n_{\text {Estanques Crocodylus }}=25 ; n_{\text {Estanques testudines }}=27\right)$; se procedió a serotipificar los aislamientos por el método convencional de Kaufmann-White y se realizaron pruebas de sensibilidad a antimicrobianos por la técnica de Kirby Bauer. Resultados. Se determinó la presencia de Salmonella sp., en un 33\% del total de estanques muestreados. El $29 \%$ de los aislamientos de Salmonella sp. serotipificados, correspondió al serogrupo B; los serogrupos C, C1, C2 y D1 presentaron menores porcentajes. Con las pruebas de sensibilidad a antimicrobianos se determinó que el $100 \%$ de los aislamientos fueron sensibles a norfloxacina. Conclusiones. La ocurrencia de Salmonella sp., en los estanques de la EBTRF fue del 33\%, con la mayor presencia del serogrupo B, donde se encuentran especies con características ampliamente zoonóticas. Con los resultados obtenidos es necesario el seguimiento de las normas de bioseguridad establecidas en la estación para el manejo de las poblaciones allí mantenidas y evitar de esa manera la ocurrencia de cuadros zoonóticos.

Palabras clave: Crocodylus intermedius, testudines, Salmonella sp., serotipificación, zoonosis. (Fuente: AIMS). 


\section{ABSTRACT}

Objective. To determine the presence of Salmonella sp., in aquatic environment of Crocodylus intermedius and testudines at the Estación de Biología Tropical Roberto Franco (EBTRF). Materials and methods. Standard methodology was used to isolate Salmonella sp., from water and sediment samples of 52 ponds $\left(n_{\text {ponds crocodylus }}=25 ; n_{\text {ponds testudines }}=27\right)$. Salmonella, isolates were serotyped by the Kaufmann-White conventional method and tested for antimicrobial susceptibility by Kirby Bauer technique. Results. The presence of Salmonella sp., was found in 33\% of the ponds checked, of which $29 \%$ corresponded to serogroup B. Serogrups C, C1, C2 and D1 were detected in a lower percentage. By means of the antimicrobial susceptibility test, $100 \%$ of isolates were sensitive to norfloxacin. Conclusions. The predominant presence of Salmonella sp., at EBTFF ponds, corresponded to serogroup B (33\%), including species of widely zoonotic characteristics. According to the results, it is necessary to establish biosafety standards at the Estación de Biología Tropical Roberto Franco to avoid zoonotic problems.

Key words: Crocodylus intermedius, testudines, Salmonella sp., serotyping, zoonoses. (Source: AIMS).

\section{INTRODUCCIÓN}

La Estación de Biología Tropical Roberto Franco (EBTRF), ubicada en la ciudad de Villavicencio - Meta, es un centro de recuperación de fauna silvestre, especialmente reptiles en amenaza $\mathrm{y} / \mathrm{o}$ vía de extinción, que con el manejo de dichas poblaciones logra minimizar el riego de extinción de las especies (Tabla 1)(1); su labor se encamina al cuidado y recuperación de ejemplares de Crocodylus intermedius y Testudines, allí se llevan a cabo investigaciones en el ámbito reproductivo que permiten la repoblación de estos ejemplares.

La Salmonella sp., es un microorganismo perteneciente a la familia Enterobacteriacea, un reconocido patógeno de mamíferos, pero que hace parte de la flora comensal del tracto gastrointestinal de los reptiles, por lo que éstos son considerados como portadores asintomáticos; sin embargo, puede comportarse como oportunista y colonizarlos también sistémicamente teniendo como vía de entrada el agua, el suelo, el alimento contaminado $\mathrm{y} / \mathrm{o}$ mediante transmisión vertical (2).

La susceptibilidad de los reptiles a patologías causadas por enterobacterias especialmente del género Salmonella $s p$, se relacionan con estados de inmunodepresión en el animal, lo que se puede producira causa del estrés por cautiverio, a la alteración en temperaturas medioambientales, al número de microorganismos presentes, la edad y el estado nutricional del animal entre otras (2-5). Cuando estas afecciones se presentan el resultado es la alteración de tejidos y fluidos por la generación de coagulación intravascular diseminada, edema, ulceraciones, necrosis, anemia, disminución de la actividad, pérdida de peso progresiva, anorexia, polifagia, regurgitación, diarrea, constipación y letargia (3).

Aunque son escasos los reportes acerca de la presencia de Salmonella sp. en estas especies silvestres y específicamente en Crocodylus intemedius, se ha establecido que su presencia es constante, por lo que se deben conocer las serovariedades implicadas y la posible susceptibilidad que tienen tanto de los animales cautivos como de los salvajes, puesto que parece ser que en los centros donde los reptiles están en condiciones de cautiverio, hay mayor posibilidad de aislamiento de esta bacteria que en estado libre $(6,7)$. Con base en lo anterior, es necesario tener en cuenta que el conocimiento de la presencia 
de patógenos tanto en el hábitat como en la población microbiana cloacal de estas especies, es fundamental para diagnosticar desórdenes en la flora digestiva, así como para prevenir infecciones secundarias que puedan poner en peligro la sobrevivencia de los ejemplares (8), todo ello dirigido a optimizar los respectivos programas de conservación.

Reconociendo la transmisión de Salmonella sp., por agua y teniendo en cuenta la importancia clínica especialmente en animales inmunosuprimidos, se llevó a cabo el aislamiento e identificación de enterobacterias del género referido, a partir de muestras del agua y del sedimento de los estanques donde se encuentran los ejemplares, además se establecieron los serogrupos que predominan en dichas especies.

A pesar de no ser uno de los objetivos planteados, durante la realización de este estudio se realizó una observación sobre la condición general de los ejemplares de Crocodylus intermedius y Testudines cautivos en la EBTRF, debido a que de acuerdo con las listas oficiales de especies amenazadas publicadas por la Unión Internacional para la Conservación de la Naturaleza (UICN), son los reptiles las especies de vertebrados más amenazadas en Colombia $(1,9)$; vale la pena aclarar que no sé realizó examen clínico a fin de evitar un "estrés" adicional por la manipulación de los ejemplares.

El objetivo del presente estudio fue determinar la presencia de microorganismos del género Salmonella sp., en el ambiente acuático de los ejemplares Crocodylus intermedius y Testudines en la Estación de Biología Tropical Roberto Franco (EBTRF).

\section{MATERIALES Y MÉTODOS}

Análisis de datos. Los datos obtenidos fueron analizados mediante estadística descriptiva y su interpretación se facilitó con la elaboración de tablas, histogramas y gráficos circulares, entre otros.
Sitio de estudio. Esta investigación se llevó a cabo en la EBTRF ubicada en la ciudad de Villavicencio Meta, centro de investigación de la Facultad de Ciencias de la Universidad Nacional de Colombia Sede Bogotá.

Las condiciones climáticas muestran un régimen de lluvias unimodal con una precipitación media anual de $4085 \mathrm{~mm}$; temperatura promedio de $27^{\circ} \mathrm{C}$ y una humedad relativa de $77.8 \%(9)$.

La EBTRF, cuenta actualmente con una colección viva que supera los 300 ejemplares de tortugas terrestres nativas del país, distribuidas en más de 20 especies (Tabla 1) y adicionalmente un número mayor a 200 ejemplares de Crocodylus intermedius distribuidos en diferentes grupos etáreos entre los que se encuentra una gran cantidad ( $80 \%)$ de neonatos y juveniles, producto de investigaciones en el área reproductiva.

Población muestreal. El muestreo se realizó sobre la totalidad de estanques $(\mathrm{N}=52)$ de la Estación. El total de animales muestreados en forma indirecta fue de 29 caimanes adultos (4 años en adelante) distribuidos en 8 estanques, 118 ejemplares juveniles (3-4 años) ubicados en 8 estanques y 80 caimanes neonatos (1-2 años) distribuidos en 9 estanques $\left(n_{\text {EstanquesCrocodylus }}=25\right)$. Se muestrearon un total de 342 individuos del Orden Testudinea distribuidos en 19 especies (Tabla 1) de las cuales 14 se encuentran en diferentes grados de riesgo de extinción (1); cabe resaltar que dichos ejemplares se encontraban ubicados en 27 estanques $\left(n_{\text {Estanques testudines }}=27\right)$.

A pesar de haberse realizado un muestreo indirecto con el fin de evitar el estrés por captura, fue evidente que todos los ejemplares se encontraban clínicamente sanos. En la EBTRF, no se han presentado brotes epidemiológicos de salmonelosis en los ejemplares.

Muestreo. Se realizaron tres muestreos seriados con un rango de diferencia de 15 días cada uno, dada la característica 
Tabla1. Ejemplares colección viva EBTRF

\begin{tabular}{|c|c|c|c|c|c|}
\hline & \multicolumn{2}{|c|}{ Especies } & \multirow{2}{*}{$\begin{array}{l}\text { No Estques } \\
\text { Muestreados }\end{array}$} & \multirow{2}{*}{$\begin{array}{l}\text { No Ejempls } \\
\text { Muestreados }\end{array}$} & \multirow{2}{*}{ Grado de Riesgo (1) } \\
\hline & Nombre Científico & Nombre Común & & & \\
\hline \multirow[t]{2}{*}{ Caimanes } & Crocodylus intermedius & $\begin{array}{l}\text { Caimán del Orinoco / } \\
\text { Caimán Llanero }\end{array}$ & 25 & 227 & Peligro Crítico \\
\hline & Caiman crocodylus & Babilla & 1 & 2 & Preocupación menor \\
\hline \multirow[t]{19}{*}{ Tortugas } & & & 27 & 342 & \\
\hline & Rhinoclemmys melanosterna & Palmera & 2 & 72 & Casi amenazado \\
\hline & Rhinoclemmys diademata & Inguensa & 2 & 32 & Vulnerable \\
\hline & Rhinoclemmys funerea & & & & Sin Peligro \\
\hline & Rhinoclemmys nasuta & Sabaletera & 1 & 2 & Datos insuficientes \\
\hline & Híbrido Rhinoclemmys & Híbrido & 2 & 115 & Sin peligro \\
\hline & Geochelone denticulata & Morroco Amar & 1 & 4 & En peligro / Vulnerable \\
\hline & Geochelone carbonaria & Morroco Negro & 1 & 15 & Peligro crítico \\
\hline & Phrynos gibbus & Hedionda & 1 & 4 & Sin Peligro \\
\hline & Phrynos geoffroanus & Bachala & 1 & 1 & Sin peligro \\
\hline & Kinosternon sp. & Tapaculo & 1 & 3 & Vulnerable \\
\hline & Peltocephalus dumerilianus & Cabezon & 1 & 1 & Casi amenazado \\
\hline & Chelus fimbriatus & Mata Mata & 1 & 2 & Casi amenazado \\
\hline & Podocnemis unifilis & Terecay & 1 & 20 & En peligro / Peligro crítico \\
\hline & Podocnemis vogli & Sabanera & 1 & 26 & Casi amenazada \\
\hline & Podocnemis expansa & Charapa & 1 & 8 & En peligro / Peligro crítico \\
\hline & Podocnemis lewyana & Lewyana & 1 & 2 & En Peligro \\
\hline & Trachemys scripta ornata & Pecho carey & 1 & 3 & Casi Amenazada \\
\hline & Trachemys scripta callirostris & Icotea & 1 & 31 & Vulnerable \\
\hline
\end{tabular}

de excreción intermitente de la bacteria por parte del animal (10-13). En caso de reportarse la presencia de Salmonella sp., el estanque positivo fue excluido para el siguiente muestreo y los estanques negativos se muestrearon nuevamente de acuerdo con lo programado. Durante este periodo se suspendió el recambio de agua de los estanques.

\section{Recolección de muestra de agua y sedimento de los estanques.}

Crocodylus intermedius neonatos y testudines. Una vez se homogenizó por agitación el agua de los estanques, se introdujo una jeringa estéril de $50 \mathrm{ml}$ a la cual previamente se le había conectado una sonda estéril de $50 \mathrm{~cm}$ de longitud y se realizó aspirado del fondo del estanque obteniendo una muestra total de $250 \mathrm{ml}$ de agua y sedimento, esta se depositó en bolsas de cierre hermético. Se empleó una jeringa y una sonda por cada estanque.

Crocodylus intermedius adultos y juveniles. La toma de muestras se realizó a partir de los canales de desagüe, se abrieron los sistemas de evacuación y se tomaron aproximadamente $250 \mathrm{ml}$ de agua y sedimento directamente en la bolsa de cierre hermético.

Todas las muestras se remitieron debidamente identificadas y fueron transportadas en refrigeración $\left(4^{\circ} \mathrm{C}\right)$ por un periodo menor de 6 horas (14-16) para inmediatamente ser procesadas en el laboratorio de microbiología veterinaria de la Universidad Nacional de Colombia Sede Bogotá.

Aislamiento e identificación de Salmonella sp. El cultivo de la bacteria fue realizado de acuerdo con la norma ISO 6579:2002/Amd 1:2007, con adición de algunos medios de cultivo que han demostrado dar buenos resultados en la recuperación del microorganismo (17-19). Las muestras fueron procesadas para evaluar la presencia de Salmonella sp., siguiendo cuatro pasos en el procedimiento:

Pre-enriquecimiento no selectivo. A cada muestra se le adicionaron $250 \mathrm{ml}$ de caldo lactosado estéril e inmediatamente se llevó a incubación a una temperatura de $37^{\circ} \mathrm{C}$, durante un periodo de $18-24$ horas $(14,17)$. 
Enriquecimiento selectivo. A partir del caldo de pre-enriquecimiento no selectivo, se obtuvo con una jeringa estéril $10 \mathrm{ml}$ de la suspensión y se llevó a un tubo de ensayo con $10 \mathrm{ml}$ de caldo tetrationato que se incubó en baño serológico a $43 \pm 0.5^{\circ} \mathrm{C}$ por $24 \mathrm{~h}$ (14).

Aislamiento en medios selectivos $y$ diferenciales: Se evaluaron dos medios de cultivo selectivos con diferentes fundamentos de identificación: Agar MacConkey (Mck) ${ }^{\mathrm{BD} \otimes}$ y CHROMagar Orientación ${ }^{\mathrm{BD} \otimes}$, la siembra se realizó mediante técnica de aislamiento o agotamiento de colonias; las cajas se incubaron a una temperatura de $37^{\circ} \mathrm{C}$ durante $24 \mathrm{~h}$. Transcurrido este tiempo, las colonias compatibles con el género Salmonella sp. se detectaron en agar MacConkey ${ }^{\mathrm{BD} \otimes}$ por su característica macroscópica de ser lactosa negativo y en CHROMagar Oientación ${ }^{\mathrm{BD}}{ }^{\circledR}$ por ser incoloras debido a que no se presenta reacción con los sustratos cromógenos del medio; adicionalmente, en los medios empleados las colonias presentaban borde definido, tamaño aproximado de 1 a $1.5 \mathrm{~mm}$ de diámetro y ligeramente convexas $(18,19)$.

\section{Identificación preliminar. Las} colonias compatibles de Salmonella sp. fueron aisladas en medios diferenciales como Agar Hektoen Entérico (H.E) $(17,18)$ y CHROMagar Salmonella ${ }^{B D}{ }^{\circ}$. Macroscópicamente en H.E las colonias se clasificaban como sospechosas de
Salmonella sp. por su característica lactosa negativo evidenciándose en ella una coloración verde - azulada con precipitado negro en el centro debido a la producción de sulfuro de hidrógeno, lo que le da la apariencia de colonia en "ojo de pescado". En CHROMagar SalmonellaBD® debido a diferencias metabólicas en la presencia de cromógenos del medio, las colonias de Salmonella sp. se observan de color rosa - púrpura, mientras que los microorganismos pertenecientes a otros géneros se observan con pigmento verde - azulado o son completamente inhibidos (Figura 1) (18).

Identificación bioquímica. El reconocimiento definitivo de los microorganismos pertenecientes al género Salmonella $s p$. se realizó mediante el sistema de identificación $\mathrm{BD}$ BBL Crystal ${ }^{\mathrm{BD} \otimes}$ para bacterias entéricas/no fermentadoras siguiendo las indicaciones del productor $(20,21)$.

Serotipificación. Los aislamientos de Salmonella $s p$. fueron serotipificados hasta antígeno somático con antisueros polivalentes específicos ( $B$ y $C$ ) y antisueros monovalentes de grupo ( $B, C 1, C 2$ y $D 1)$, se identificaron de acuerdo al esquema de Kauffman - White $(2,22)$.

Susceptibilidad a antibióticos. La prueba de sensibilidad a antimicrobianos se realizó mediante el método de Kirby Bauer. Los antibióticos evaluados fueron

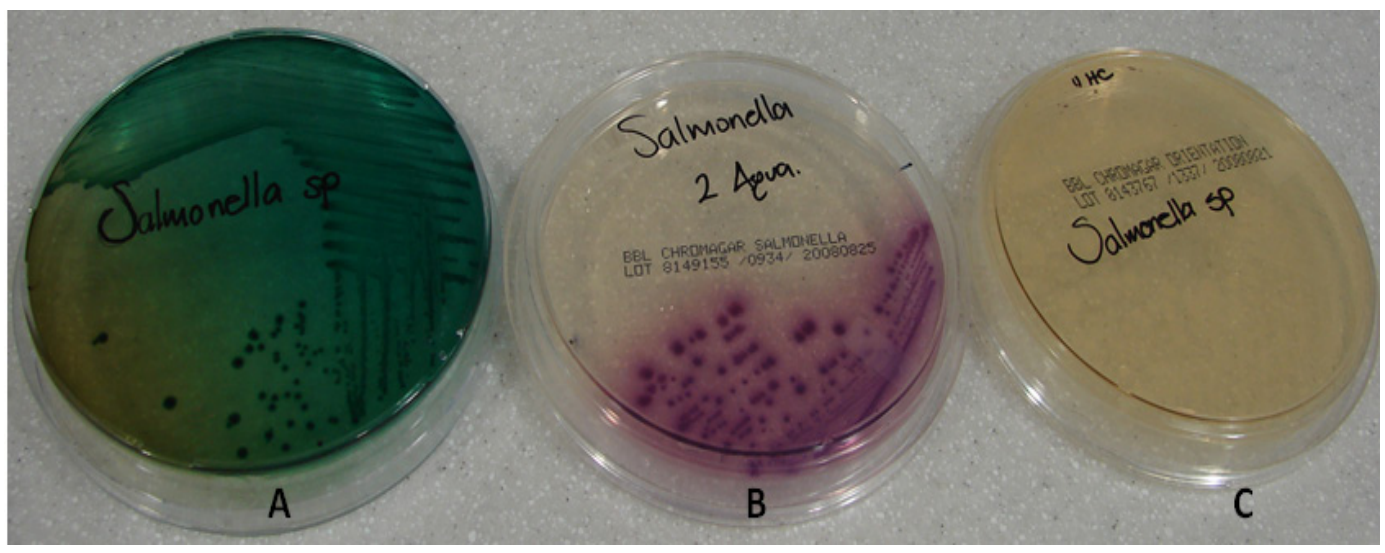

Figura 1. Identificación de colonias compatibles con Salmonella sp. en medios de cultivo selectivos y diferenciales (A. Agar Hektoen, B. CHROMagar Salmonella ${ }^{B D}$ y C. CHROMagar Orientación $\left.{ }^{\mathrm{BD}}{ }^{\circledR}\right)$. 
Sulfatrimetoprim $25 \mu \mathrm{g}$, Norfloxacina 10 $\mu \mathrm{g}$, Ampicilina $10 \mu \mathrm{g}$, Cloranfenicol $20 \mu \mathrm{g}$, Cefoxitin $20 \mu \mathrm{g}$, y Tetraciclina $30 \mu \mathrm{g}(10,18)$.

\section{RESULTADOS}

Positividad a Salmonella sp. en Estanques de Crocodylus y Testudines. Los $\mathrm{N}=52$ estanques de la EBTFF, se encuentran distribuidos entre las especies de Crocodylus de diferentes grupos etáreos ( $\mathrm{n}_{\text {Estanques Crocodylus }}=25$, así: 1 con Caiman Crocodylus (Babillas) y 24 con Crocodylus intermedius) y 27 estanques de Testudines $\left(\mathrm{n}_{\text {Estanques Testudines }}=27\right)$.

En la figura 2 se encuentran consignados los resultados obtenidos en la totalidad de estanques, donde se estableció que en 17 (33\%) de los 52 estanques muestreados

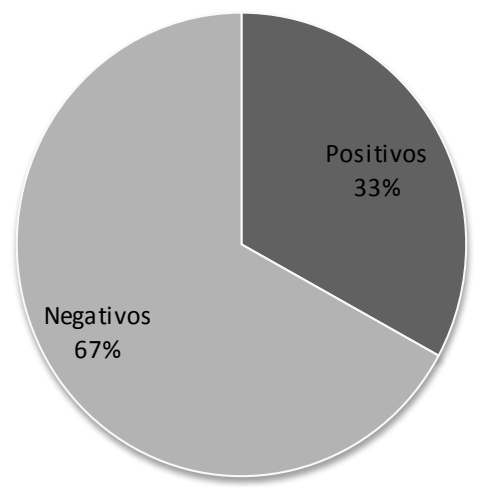

Figura 2. Estanques positivos para el aislamiento de microorganismos del género Salmonella sp. $(N=52)$

fue posible recuperar microorganismos pertenecientes al género Salmonella sp. mientras los 35 (67\%) restantes se encontraban negativos para la bacteria.

En 9 de los 24 estanques con Crocodylus intermedius se hallo Salmonella sp., y el estanque ocupado con ejemplares de Caiman crocodylus (Babillas) fue igualmente positivo a la bacteria, lo que representa el $40 \%(n=10)$ de positividad. Por otro lado, de los 27 estanques de tortugas, 7 (25.9\%) fueron positivos para Salmonella sp., encontrándose la presencia del microorganismo en 7 de las 18 especies

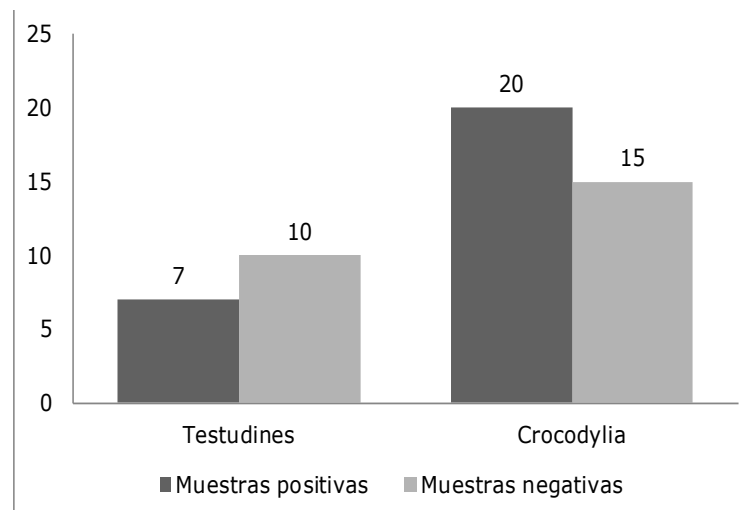

Figura 3. Muestras positivas y negativas para el aislamiento de Salmonella sp., según orden.

que conforman la colección viva de la EBTRF. (Figura 3)

Es necesario aclarar que en el primer muestreo se remitieron al laboratorio 52 muestras de agua y sedimento, en el segundo muestreo 37 y en el tercero 36 muestras; esto debido a que si en alguno de los estanques evaluados se reportaba la presencia de Salmonella sp., no era relevante muestrear nuevamente dicho estanque ya que el estudio estaba condicionado a la evaluación de la presencia/ausencia de la bacteria

Serotipificación. Los 17 aislamientos identificados por el método de Crystal ${ }^{\mathrm{BD}}{ }^{\circledR}$ como Salmonella sp. se lograron clasificar con antisueros comerciales DIFCO $^{\circledR}$, hallando 6 serogrupos prevalentes en la Estación. En la figura 4 se observa que de los

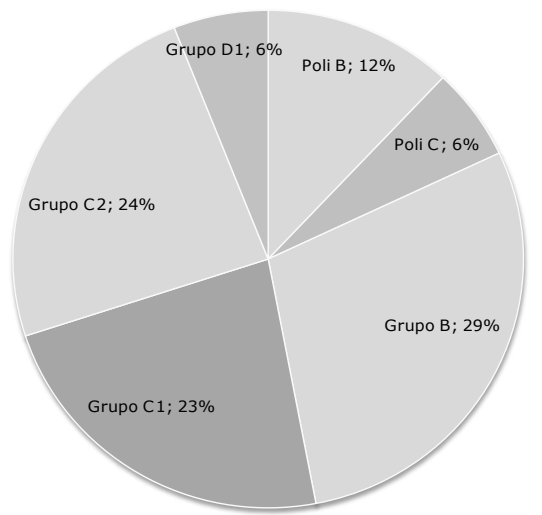

Figura 4. Serogrupos identificados hasta antígeno somático de las cepas aisladas en las muestras de agua-sedimento de los estanques de la E.B.T.R.F 
aislamientos de Salmonella sp., hallados en las muestras de agua y sedimento, el $29 \%$ pertenecen al serogrupo $B$; mientras que la presencia de las bacterias clasificadas como pertenecientes al serogrupo $\mathrm{C} 1$ y serogrupo C2 fue de 23 y $24 \%$, respectivamente. El porcentaje restante de los aislamientos pertenecen a serovariedades incluidas en los serogrupos que abarca el antisuero polivalente B $(12 \%)$, serogrupo D1 (6\%) y serogrupos pertenecientes al polivalente C $(6 \%)$

Pruebas de sensibilidad a
antimicrobianos. Los resultados
obtenidos en las pruebas de sensibilidad a
partir de los aislamientos de Salmonella sp.
provenientes de Crocodylus y testudines,
se encuentran consignados en la tabla 2 y
en la figura 5, donde se puede observar

Tabla 2. Comportamiento de microorganismos del género Salmonella sp., frente a antibióticos.

\begin{tabular}{lcccccc}
\hline & \multicolumn{2}{c}{ Sensible (n) } & \multicolumn{2}{c}{ Moderado (n) } & \multicolumn{2}{c}{ Resistente (n) } \\
\hline \multirow{2}{*}{ Ampicilina } & $T^{*}$ & $\mathrm{C}^{* *}$ & $\mathrm{~T}^{*}$ & $\mathrm{C}^{* *}$ & $\mathrm{~T}^{*}$ & $\mathrm{C}^{* *}$ \\
Cefoxitin & 3 & 8 & - & 2 & 4 & - \\
Cloranfenicol & 3 & 8 & - & 1 & 4 & 1 \\
Norfloxacina & 3 & 10 & - & - & 4 & - \\
Sulfatrimetoprim & 7 & 10 & - & - & - & - \\
Tetraciclina & 3 & 9 & - & - & 4 & 1 \\
\hline
\end{tabular}

*Testudines, **Crocodylia

- Sensible " Sensibilidad Moderada $\square$ Resistente

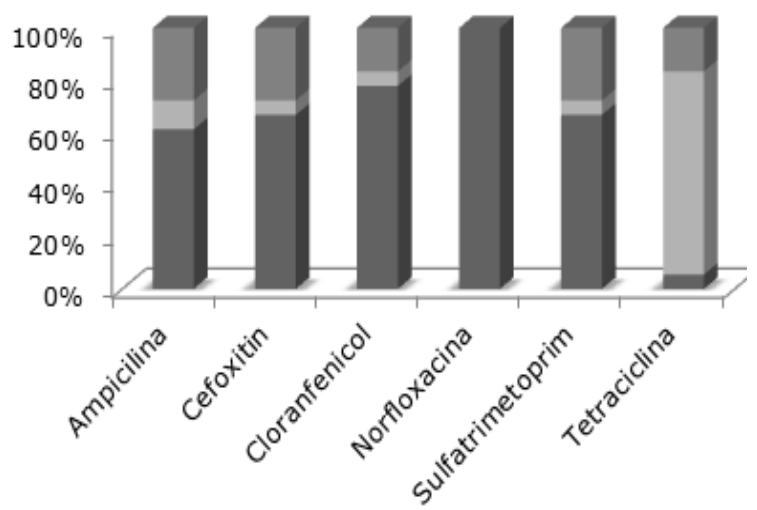

Figura 5. Porcentaje de sensibilidad, resistencia y sensibilidad moderada de aislamientos de Salmonella sp. a partir de las muestras de agua-sedimento de Crocodylus y Testudines en la EBTFF. el comportamiento de la bacteria ante diferentes antibióticos.

Se puede establecer que existen diferencias notables para cada una de las especies, puesto que las Salmonella sp., obtenidas de muestras provenientes de caimán son mucho más sensibles a los antimicrobianos que las de testudines, donde varios de los aislamientos fueron resistentes a los antibióticos utilizados; por ejemplo, para cloranfenicol todas las cepas provenientes de caimán fueron sensibles mientras que en testudines 4 evidenciaron resistencia al antibiótico.

\section{DISCUSIÓN}

Es de vital importancia tener en cuenta, que es muy poco lo que se ha investigado a nivel mundial acerca de la presencia de la Salmonella sp. en especies como Crocodylus intermedius, que se encuentran en peligro crítico de extinción (1) y que otros reptiles son el principal objetivo de los centros de conservación y mantenimiento de las especies mediante programas de reproducción. Por muchos años se han encaminado estudios hacia especies que son criadas como mascotas no convencionales o exóticas, tales como iguanas, lagartos, tortugas y serpientes $(8,23-28)$, pero no en animales con algún grado de amenaza o en vía de extinción; adicionalmente las investigaciones acerca de la presencia de Salmonella sp. en los animales y en su hábitat natural son insuficientes.

Si bien es cierto que Salmonella sp. hace parte de la flora normal intestinal de los reptiles y que su aislamiento en testudines de diferentes familias ha sido reportado con mayor frecuencia $(8,23,25,26-29)$ que en Crocodylus; no se puede ignorar que para todos los individuos evaluados tanto tortugas como caimanes, las condiciones de cautiverio donde son mantenidos crean cuadros de inmunosupresión marcada donde la enterobacteria es excretada de manera intermitente $(10-13,30,31)$ y que puede llegar a colonizar el tracto digestivo hasta producir cuadros patológicos con estados de letargia, anorexia, septicemia, neumonía, 
celomitis, abscesos, shock hipovolémico y bajo circunstancias extremas, la muerte del ejemplar (32-34).

Aunque todos los reptiles son considerados portadores naturales de Salmonella sp., lagartos, serpientes y tortugas en particular, son reservorios de esta bacteria $(23,24,35)$; sin embargo, no es claro como estas especies son colonizadas de forma natural por serovariedades de Salmonella sp. Respecto de la transmisión, en algunos estudios se ha evaluado la capacidad de colonización de la bacteria en útero, contaminación perinatal, por ingestión de presas contaminadas y/o contacto con heces de otros reptiles $(32,36)$; según Kourany y Telford (33), la predisposición a condiciones de estrés y escases de alimento hacen que la bacteria atraviese la barrera sanguínea y linfática llegando a oviducto, causando así la contaminación de los huevos; sin embargo, Mitchell y Shane (26), sugieren que la contaminación de los huevos se da por abrasión del mismo cuando entra en contacto con el suelo y cáscaras remanentes, descartando así la transmisión vertical puesto que no lograron el aislamiento del microorganismo a partir de ovarios, oviducto y saco embrionario.

Respecto de la transmisión horizontal, la mayoría de investigaciones concluyen que la ingesta de agua contaminada con heces es la principal vía de infección por el ambiente, considerándose el medio acuático favorable para ello (31) sin llegar a subestimar que el alimento y el contacto con otros reptiles también son una importante vía de transmisión. Las altas tasas de ejemplares portadores de Salmonella sp., se relacionan con el comportamiento de coprofagia por parte de las crías, lo que a su vez asegura la transmisión prematura de dichos microorganismos (3).

De acuerdo con algunos estudios, las muestras de origen ambiental permiten un mayor porcentaje de recuperación del microorganismo dado que la bacteria no es excretada de manera continua; realizar únicamente muestreos de hisopado cloacal no asegura la obtención de resultados positivos puesto que no es posible determinar si el ejemplar en estudio está excretando la bacteria en el momento de la recolección de la muestra $(9,36)$. Salmonella sp. es altamente resistente fuera de su hospedero y permanece viable después de 89 días en el suelo y/o agua y 30 meses en materia fecal, lo que permite inferir que todo el ambiente del encierro es fuente para aislamientos positivos para la enterobacteria en mención (30).

La resistencia a antimicrobianos por parte de los aislamientos de Salmonella sp. a partir de muestras de origen animal es un problema emergente; estas bacterias pueden ser una fuente de plásmidos de resistencia para otras bacterias y producir alteraciones en el tratamiento antimicrobiano clave para combatir enfermedades en medicina humana y veterinaria $(37,38)$. Simultáneamente se puede asegurar que el antibiótico de elección para tratar casos sintomáticos en la EBTRF es norfloxacina debido a que todos los aislamientos de Salmonella sp. enfrentados a este antimicrobiano tienen un alto grado de sensibilidad (Figura 5); de igual manera se establece que tetraciclina tiene un comportamiento de sensibilidad intermedia para todos y que cloranfenicol es un antimicrobiano que tendría alto rendimiento contra los microorganismos de este género obtenidos a partir de ejemplares de caimán; sin embargo, dicho antibiótico tiene restricciones al ser suministrado por generar alteraciones y degeneraciones a nivel de médula ósea (9).

No se puede olvidar que el uso de antibióticos de amplio espectro como las quinolonas (norfloxacina) que mostraron un $100 \%$ de efectividad frente a los aislamientos de la estación (Figura 5), son ampliamente utilizados en medicina herpetológica y pueden ser causa de alteración momentanea grave de la flora Gram negativa y Gram positiva que se encuentra en el tracto gastrointestinal de estas especies; existen datos bibliográficos que demuestran una cierta tendencia a la anorexia o a alteraciones digestivas en reptiles herbívoros tras terapias prolongadas de antibióticos (39).

A la importancia médica de las infecciones por Salmonella sp. en centros de conservación/ zoocría y en el hábitat natural, se le suma el 
potencial zoonótico, ya que los aislamientos de la bacteria a partir de reptiles se han considerado como patógenos para humanos, al poseer factores de patogenicidad cruciales en el desarrollo de una gastroenteritis (20).

Los serogrupos de Salmonella sp. hallados en agua y sedimento de los estanques de la EBTRF han sido frecuentemente reportados en reptiles; teniendo en cuenta que el grupo $B$ fue el serogrupo de mayor ocurrencia (29\%) y el serogrupo D1 fue hallado en un $6 \%$, es posible inferir en la hipótesis de encontrar serovariedades con alto potencial zoonótico, puesto que en estos serogrupos se encuentran incluidas especies como Salmonella typhimurium (Grupo B) y $S$. enterididis (Grupo D), dos de los serovares comunmente implicados en aproximadamente el $50 \%$ de los casos de salmonelosis en personas de los Estados Unidos (33) , lo que permite plantear que el personal que labora en la Estación se encuentra en riesgo de contraer el patógeno.

Adicionalmente, según Popoff y Le Minor (12) y Grimont y Weill (13), en el serogrupo C2 se encuentran incluidas serovariedades como S. newport y $S$. muenchen que son agentes causales de salmonelosis en humanos, reptiles y caprinos, entre otras especies $(12,13,26$, 28). Por otra parte, los antisueros polivalentes B y C, incluyen serovariedades de Salmonella que han sido aisladas constantemente a partir de muestras de reptiles, allí se encuentran S. oranienburg, S. berta, S. panama, $S$. rubislaw, entre otras; muchas de las cuales se han reportado como agentes etiológicos de patologías gastrointestinales en los reptiles y algunas veces asociadas a salmonelosis en humanos, producidas por contacto directo con el animal asintomático $(5,17,18,24)$.

Cabe resaltar, que aún sin la serotipificación completa de los aislamientos de Salmonella sp. obtenidos en la EBTRF, es pertinente considerar que el personal que labora allí se encuentra en inminente riesgo de contraer salmonelosis, por lo tanto, como método de prevención es necesario el seguimiento de las normas de bioseguridad establecidas para el manejo de las poblaciones allí mantenidas.
Este documento es el primer reporte sobre microbiología del ambiente en ejemplares de Crocodylus intermedius en Colombia, siendo de importancia para la medicina de reptiles y aportando al conocimiento del riesgo zoonótico en el personal a cargo de este tipo de ejemplares.

En conclusión, se logró aislar e identificar mediante análisis microbiológicos la presencia de enterobacterias del género Salmonella sp., en muestras de agua-sedimento de estanques con ejemplares de Testudines y Crocodylus mantenidos en cautiverio en la Estación de Biología Tropical Roberto en la Ciudad de Villavicencio; estableciendo su ocurrencia en el $33 \%$ de los estanques.

Los ejemplares de la EBTRF son portadores asintomáticos de Salmonella sp.; sin embargo, se deben implementar buenas prácticas de manejo en los animales de manera que se minimice el potencial patógeno oportunista del microorganismo y se evite el desencadenamiento de cuadros clínicos asociados con salmonelosis en los reptiles.

La norfloxacina es el antibiótico ideal para tratar los ejemplares de la Estación Roberto Franco que puedan presentar sintomatología asociada a salmonelosis, aunque su administración debe ser cuidadosa con el fin de evitar alteraciones en la flora digestiva natural de los reptiles.

La presencia de Salmonella sp. en el ambiente, hace necesario el seguimiento de las normas de bioseguridad establecidas en la Estación para el manejo de las poblaciones allí mantenidas y evitar de esa manera la presentación de cuadros zoonóticos.

\section{Agradecimientos}

Al Laboratorio de Microbiología de la Facultad de Medicina Veterinaria y Zootecnia de la Universidad Nacional de Colombia. A Becton Dickinson Colombia Ltda. por la dotación de medios de cultivo, kit de identificación y sensidiscos. Al equipo humano de la Estación de Biología Tropical Roberto Franco por toda su colaboración y especialmente a su directora profesora María Cristina Ardila por sus aportes al trabajo. 


\section{REFERENCIAS}

1. Castaño O V. Libro rojo de reptiles de Colombia. Serie Libros Rojos de Especies Amenazadas de Colombia. Instituto Nacional de Ciencias Naturales - Universidad Nacional de Colombia y Ministerio del Medio Ambiente. 2002. Bogotá, Colombia. En: http://www. humboldt.org.co/conservacion/reptiles_ amenazados.htm

2. Popoff $M$ and Le Minor L. Antigenic Formulas of the Salmonella Serovars. 7th revisión. WHO Collaborating center for reference and research on Salmonella. Paris, France: Institut Pasteur; 1997.

3. Mader D. Reptile Medicine and Surgery. W.B. Phyladelphia, USA: Saunders Company; 1996.

4. Chiodini R J and Sundberg J P. Salmonellosis in reptiles: a review. Am J Epidemiol 1981; 113:494-499

5. Onderka $\mathrm{D} \mathrm{K}$ and Finlayson $\mathrm{M}$ C. Salmonellae and salmonellosis in captive reptiles. Can J Comp Med 1985; 49:268-270.

6. Jertborn $M$, Hagling $P$, Iwarson $S$ and Svennerholm M. Estimation of symptomatic and asymptomatic Salmonella infections. Scand J Infect Dis 1990; 22:451-455.

7. Burnham B R, Atchley D H, DeFusco $\mathrm{R} P$, Ferris $K \mathrm{E}$, Zicarelli J C, Lee J H and Angulo $F$ J. Prevalence of fecal shedding of Salmonella organisms among captive green iguanas and potencial public health implications. J Am Vet Med Assoc 213, 48-50. 1998.

8. Ramsay E C, Daniel G, Tryon B W, Merryman J I, Morris $P$ J and Bemis D A. Osteomyelitis Associated whit Salmonella enterica ss arizonae in a colony of ridgenose rattlesnakes (Crotalus Willardi). J Zoo Wildl Med 2002; 33(4):301-310.
9. Saelinger $\mathrm{C}$ and Lewbart G. Prevalence of Salmonella spp in cloacal, fecal, and gastrointestinal mucosal samples from wild North American turtles. JAVMA 2006; 229:266-273.

10. Ebani V V, Cerri D, Fratini F, Meille N, Valentini P and Andreani E. Salmonella entérica isolates from faeces of domestic reptiles and a study of their antimicrobial in vitro sensitivity. Vet Sci 2005; 78:117-121.

11. Minette H P. Epidemiologic Aspects of salmonelosis in reptiles, amphibians, mollusks and crustaceans. A review. Int J Zoonoses 1984; 11:95-104.

12. Sanyal D, Douglas $T$ and Roberts R. Salmonella infection acquired from reptilian pets. Arch Dis Child 1997; 77:345-346.

13. Ward L. Salmonella perfils pet reptiles. Commun Dis Public Health 2000; $3(1): 2-3$.

14. Rodríguez M y Ramírez J. Crocodylus intermedius (Caimán llanero) ex situ en la Estación de Biología Tropical Roberto Franco (EBTRF), Colombia. En: Velasco A, Colomine G, Villarroel G y Quero M (Eds). Memorias del taller para la conservación del caimán del Orinoco (Crocodylus intermedius) en Colombia y Venezuela. Colombia. 2000: 78-132.

15. Mitchell M A and Shane S M. Preliminary findings of Salmonella spp., in captive green iguanas (Iguana iguana) and their environment. Prev Vet Med 2000; 45:297-304

16. Shane S M, Gilbert $R$ and Harrington $\mathrm{K}$ S. Salmonella colonization in comercial pet turtles (Pseudemus scripta elegans). Epidemiol Infect 1990; 105:307-316. 
17. Luna G. Manual Operativo de Análisis Microbiológicos Para Alimentos. Primera Edición. Bogotá, D.C., Colombia: Fundación Universidad de Bogotá Jorge Tadeo Lozano; 1991.

18. Corrente $M$, Madio $A$, Friendrich $K$ G, Greco G, Desario C, Taglisbue $S$, D'Incau $M$, Campolo $M$ and Buonavoglia C. Isolation of Salmonella strains from reptile faeces and comparison of different culture media. J Appl Microbiol 2004; 96:709-715.

19. Stanchi O. Microbiología Veterinaria. Primera Edición. Buenos Aires, Argentina. Editorial Intermedica. 2007.

20. Bonnie E. Isolation and identification of Salmonella from meat, poultry and egg products. In: Microbiology laboratory guidebook. 3rd ed. Food Safety and Inspection Service. Washington D.C.: Department of Agriculture; 2001.

21. BBL CrystalTM Identification Systems Enteric/Nonfermenter ID Kit. URL Disponible En: http://www.bd.com/ ds/productCenter/245000.asp

22. Grimont $P$ and Weill $F X$. Antigenic Formulas of the Salmonella Serovars. 9th revisión. World Health Organization. Collaborating center for reference and research on Salmonella. Paris, France: Institut Pasteur; 2007.

23. Pasmans $F$, Martel A, Boyen $F$, Vanderkerchove $D$ and Wybo I. Characterization of Salmonella isolates from captive lizards. Vet Microbiol 2005; 110:285-291.

24. Hidalgo J, Díaz C, Pérez N, De Frutos $\mathrm{C}$ and Herrero A. Salmonella in free living exotic and native turtles and in pet exotic turtles from SW Spain. Vet Sci 2008; 85:449-452.

25. Bradley $T$, Angulo $F$ and Mitchell $M$. Public health education on Salmonella spp. and reptiles. JAVMA 2001; 219:754-755.
26. Mitchell M A and Shane S M. Salmonella in reptiles. Seminars in avian and exotic pet medicine. Florida: Krieger Publishing Company; 2001.

27. Frye $\mathrm{F}$ L. Infectious diseases. Fungal actinomycete, bacterial, rickettsial and viral diseases. Biomedical and Surgical Aspects of Captive Reptile husbandry. Edwardsville, Krieger: Publishing Company; 1991.

28. Baümler $A \mathrm{~J}$, Tsolis $R \mathrm{M}$, Fitch $T A$ and Adams L G. Evolution of host adaptation in Salmonella enteric. Infect Immun 1998; 66: 4579-4587.

29. Schöter $M$, Roggentin P, Hofmann J, Speicher A, Laufs R and Mack D. Pet snakes as a reservoir for Salmonella enteric subsp. Diarizonae (serogroup IIIb): a prospective study. Appl Environ Microbiol 2004; 70(1):613-315.

30. Warwick C, Lambiris A, Westwood $D$ and Steedman C. Reptile related salmonellosis. J R Soc Med 2001; 94:124-126.

31. Mermin J, Hutwagner L, Vugia D, Shallow S, Daily P, Bender J, Koehler J, Marcus R and Angulo F J. Reptiles, Amphibians, and human Salmonella infections: a population based, casecontrol study. Clin Infect Dis 2004; 38:253-261.

32. Pasmans $F$, De Herdt $P$, Dewulf J and Haesebrouk F. Pathogenesis of infection with Salmonella enteric subsp. enterica serovar Muenchen in the turtle Trachemys scripta scripta. Vet Microbiol 2002; 87:315-325.

33. Kourany M and Telford S. Salmonella and Arizona infections of alimentary and reproductivetracts of panamanian lizards. Infec Immun 1982; 36:432-434.

34. Simmons A. A multiresistant Salmonella enteric serovar Newport. Veterinary Record 2002; 1:150-159. 
35. Geüe L and Löschner U. Salmonella entérica in reptiles of German and Austrian Origin. Vet Microbiol 2002; 84:79-91.

36. Hidalgo J, Díaz C and Jimenez C. Salmonella in free living terrestrial and aquatic turtles. Vet Microbiol 2007; 119:311-315.

37. Siebeling R, Neal $P$ and Granberry D. Treatment of Salmonella - Arizona infected turtle eggs with terramycin and chloromycetin by the temperature differential egg dip method. Appl Microbiol 1975; 30(5):791-79.
38. Kaufman G.E. Pharmacology, pharmacodynamics and drug dosing. In: Ackerman, C (ed), The Biology Husbandry and Health Care of Reptiles. TFH, New Jersey: 1998.

39. Jacobson $E$ R. Use of antimicrobial drugs in reptiles. J Zoo Wildl Med 1999; 4:190-199. 